\title{
Review Article \\ Efficacy of Auricular Therapy for Pain Management: A Systematic Review and Meta-Analysis
}

\author{
Chao Hsing Yeh, ${ }^{1}$ Yi Chien Chiang, ${ }^{2}$ Samuel L. Hoffman, ${ }^{1}$ Zhan Liang, ${ }^{1}$ Mary Lou Klem, \\ Wilson W. S. Tam, ${ }^{4}$ Lung-Chang Chien, ${ }^{5}$ and Lorna Kwai-Ping Suen ${ }^{6}$ \\ ${ }^{1}$ School of Nursing, University of Pittsburgh, 3500 Victoria Street, 440 Victoria Building, Pittsburgh, PA 15261, USA \\ ${ }^{2}$ Department of Nursing, Chang Gung University of Science and Technology, No. 261, Wen-hwa 1st Road, \\ Kwei-shan, Taoyuan 333, Taiwan \\ ${ }^{3}$ Falk Library, University of Pittsburgh, 200 Scaife Hall, 3550 Terrace Street, Pittsburgh, PA 15261, USA \\ ${ }^{4}$ The Jockey Club School of Public Health and Primary Care, The Chinese Hong Kong University, Hong Kong \\ ${ }^{5}$ Division of Biostatistics, University of Texas School of Public Health San Antonio Regional Campus, \\ Research to Advance Community Health Center, University of Texas Health Science Center at San Antonio Regional Campus, \\ 7411 John Smith Drive, Suite 1050 Room 505, San Antonio, TX 78229, USA \\ ${ }^{6}$ School of Nursing, Hong Kong Polytechnic University, Hung Hom, Kowloon, Hong Kong
}

Correspondence should be addressed to Chao Hsing Yeh; yehc@pitt.edu

Received 29 April 2014; Accepted 17 June 2014; Published 23 July 2014

Academic Editor: Ke Ren

Copyright (C) 2014 Chao Hsing Yeh et al. This is an open access article distributed under the Creative Commons Attribution License, which permits unrestricted use, distribution, and reproduction in any medium, provided the original work is properly cited.

Objective. The objective of this systematic review and meta-analysis was to assess the efficacy of auricular therapy by including a sham therapy control group. Methods. Relevant, randomized clinical trials (RCTs) were identified by searching medical related databases from, depending on journal, 1900 (at the earliest) to 1994 (at the latest) through May 2013. The outcome measure was a pain intensity score. Results. Twenty-two RCTs were identified and 13 RCTs were included for meta-analysis. In these studies, auricular therapy provided significant pain relief when compared to a sham or control group. The overall standardized mean differences (SMD) was $1.59(95 \%$ CI $[-2.36,-0.82])$ (13 trials, total subject numbers $=806)$, indicating that, on average, the mean decrease in pain score for auricular therapy group was 1.59 standard deviations greater than the mean decrease for the sham control. In terms of the efficacy of the different treatment methods, auricular acupressure boasts the largest strength of evidence for pain relief, followed by auricular acupuncture. Electroacupuncture stimulation did not show significant evidence for efficacy, which may be due to the small sample size (i.e., only 19 subjects were included). Conclusion. Further large-scale RCTs are needed to determine the efficacy of auricular therapy for pain.

\section{Introduction}

Pain is a highly prevalent and costly health problem in the United States. Back pain, in particular, affects at least $84 \%$ of individuals at some point during their lives $[1,2]$, and pain recurs in up to $80 \%$ of cases within 1 year [2]. The pain can occur at any age but is most prevalent during the third decade of life [2]. In the United States, back pain is the second most common cause of disability [3], the second leading cause of lost workplace productivity (after the common cold) [4], and the third most common reason for visiting a health provider [5]. These effects place an enormous burden on U.S. society and health care systems, as reflected by an estimated cost ranging from $\$ 84.1$ billion (direct cost of health care) to $\$ 624.8$ billion (indirect cost including loss of productivity) per year [6-8]. Pain in its various manifestations is also responsible for work absences, which create an enormous economic burden on individuals, families, communities, industry, and government $[1,9]$.

Analgesic pharmaceutical use is one of most common strategies for managing pain but it is associated with a variety of adverse side effects (e.g., drowsiness, constipation, dry mouth, gastrointestinal bleeding, and potential for addiction) $[10,11]$. Pharmaceutical options are currently the first and 
best choice for acute pain. However, patients with chronic or recurrent pain often develop tolerance to narcotics over time and receive diminishing relief of their pain [12]. The high prevalence of extended and chronic pain highlights the need for better pain management strategies.

Complementary and alternative medicine (CAM) therapies, especially acupuncture, offer additional options in pain management $[13,14]$. These CAM options tend to be cheaper, less invasive, and of lower risk than the second and third line conventional treatments of strong narcotics and invasive surgical procedures. Acupuncture can reduce the severity of pain, allowing for reduced doses of medications [14]. In a 2007 government survey, Americans had spent $\$ 33.9$ billion out of pocket on CAM over the previous 12 months and an estimated $\$ 11.9$ billion on visits to CAM practitioners, including acupuncturists $[15,16]$. However, acupuncture currently is not covered by the majority of U.S. health care plans.

Auricular therapy is one form of acupuncture and a well-recognized element of traditional Chinese medicine (TCM) [17]. Auricular therapy is based on long-standing tradition and was modified and updated by Dr. Paul Nogier, the "father of auriculotherapy," in the 1950s. The World Health Organization considers auricular therapy a form of microacupuncture that can affect the whole body [18]. Auricular therapy involves the relationships among the ear, energy lines (channels and meridians), and muscle regions comprising the whole body, according to a theory known as somatic reflexology. This theory posits that when a symptom or disease arises in the body, it is projected onto the ear at a regular and measureable zone $[17,19]$. The TCM model views disease as being caused by the imbalance of a person's energy or $q i$ [17]. The stimulation of auricular acupoints is, thus, intended to regulate $q i$, activate the meridians and collateral systems, and balance the $q i$ aspects of yin and yang and, in so doing, has been successful in treating a variety of health problems, including pain [17].

Types of auricular therapy include auricular acupuncture (AA), electroacupuncture stimulation (EAS), and acupressure (AP). The former two approaches include needle insertion or application of intense electrical stimulation to ear acupoints [42]. In contrast, without needles, acupressure does not usually result in strong or painful sensations. Auricular therapy is also different from traditional body acupuncture in that auricular therapy allows needles (for AA) or acupressure patches (for AP) to remain in place up to 1 month, depending on the subject's ear and skin condition and potentially extending the therapeutic period without constant and direct provider oversight. Thus, auricular therapy can reduce both the need for patients to travel to the acupuncture site and the cost of visiting a practitioner.

Studies using auricular therapy (including AA, EAS, and AP) have shown promising effects in the pain management of several conditions, including dysmenorrhea [43-45], postoperative pain [46-48], hip fracture [22], low back pain [49,50], and bone marrow aspiration [51]. A recent meta-analysis (including studies up to December, 2008) of auricular therapy for pain management comprising 17 studies, including three conducted in the U.S. $[23,26,27]$, found that auricular therapy reduces analgesic use for perioperative pain (standard mean difference $[\mathrm{SMD}]=0.54$ (95\% confidence interval (CI) $0.30,0.77)$ ) and reduces pain intensity for acute and chronic pain (SMD = $1.56(95 \%(\mathrm{CI}): 0.85,2.26)$ ) compared with control groups [52].

Studies in auricular therapy for pain management have increased since the 1980s. In order to gather and evaluate up-to-date evidence of auricular therapy efficacy for pain management, we conducted this meta-analysis, based on previous studies of systematic reviews [53] and meta-analyses [52], and expanded it to include the most current studies (up to May 30, 2013). Moreover, we included Chinese research literature in this meta-analysis because auricular therapy not only has been popular in Chinese-culture for more than 2,000 years, but also is a ubiquitous treatment for pain throughout Asia.

\section{Methods}

2.1. Data Sources and Searches. The literature search was performed using Ovid MEDLINE (1966 to May 2013), Ovid CINAHL (1982 to May 2013), Wiley Cochrane CENTRAL (1948 to May 2013), Embase.com (1980 to May 2013), Ovid AMED (1985 to May 2005), Ovid MANTIS (1900 to May 2013), ISI Web of Science (to May 2013), China Biological Medicine Database (CBM disc 1980 to May 2013), Chinese Medical Current Contents (CMCC) (1994 to May 2013), and China Academic Journals (CAJ) Full Text Databases (1994 to May 2013). The search keywords included auriculotherapy, auricular acupuncture, auricular electroacupuncture/TENS, auricular acupressure, and laser auriculotherapy (see example search in Table 2). For most database searches, topic search terms were combined with sensitive methodology filters designed to identify RCTs. Additional studies were identified through the references list in a recent article [52] reviewing studies of auricular therapy used for pain management. EndNote software was used to manage citations obtained through the database search.

2.2. Inclusion and Exclusion Criteria. In order to determine if the studies were eligible to be included in the study, two reviewers (Yi Chien Chiang and Lorna Kwai-Ping Suen) independently appraised the titles and abstracts of the English and Chinese articles. Relevant studies were retrieved, and the full articles were assessed by two independent reviewers for inclusion (Yi Chien Chiang and Lorna Kwai-Ping Suen). Any disagreement on inclusion was resolved through discussion. To be included in the analyses, the trials had to meet the following criteria: they (1) were RCTs, (2) were published in English or Chinese peer-reviewed journals, (3) compared auriculotherapy to sham and/or standard medical care with wait-list control, and (4) used a validated pain outcome measurement, including Visual Analog Scale for Pain (VAS Pain), Numeric Rating Scale for Pain (NRS Pain), or McGill Pain Questionnaire. Studies were excluded if they (1) were not RCTs, (2) combined auriculotherapy with other treatment (leading to a lack of clear evidence for efficacy), or (3) had no pain outcome measure. Recorded data included 
study characteristics, patient characteristics, inclusion and exclusion criteria, mode of treatment and control procedures, and outcomes. If more than one outcome measure was reported, separate evaluations were made for least and most favorable outcomes. Letters were sent to authors requesting information if we were not able to retrieve the data for metaanalysis from the article.

2.3. Data Synthesis and Analysis. The studies we decided to include for analysis were assessed by methodological quality (MQ) [54], which was designed for the criteriabased meta-analysis of acupuncture studies and has been accepted for use in many systematic analyses and metaanalysis of complementary therapies [55-57]. The criteria for MQ include four main categories: (1) comparability of prognosis (including method of randomization, sample size, and coverage of withdrawal and dropouts) (35 points), (2) adequate intervention (including intervention procedure, control group, and quality of the intervention) (25 points), (3) adequate effect measurement (including blinding, followup, remarks on side effects, and confounding variables) (30 points), and (4) data presentation and analysis (10 points). The maximum total score is 100 points; a score over 50 indicates a research report of good quality [54]. See Table 3 for detailed information.

In order to be included in the final meta-analysis, mean and standard deviation data from each study had to be retrieved. If the data were not available from the published manuscript, the authors were contacted to hopefully provide the data. Eight trials were excluded in the meta-analysis due to incomplete data. All pain intensity scores were continuous. Thus, standardized mean differences (SMD) and 95\% confidence intervals (CI) were calculated to compare the pain scores between the treatment and the sham/control group in each study. A magnitude effect size (SMD) of 0.2, 0.5, and 0.8 was defined as small, medium, and large, respectively [58]. Random-effects models were used to estimate the combined effect, and $\chi^{2}$ statistics were used to assess the heterogeneity. Additionally, $I^{2}$ statistics were also computed to show the percentage of variation due to heterogeneity [59]. Finally, publication bias was assessed by funnel plots.

\section{Results}

3.1. Quality Assessment. Figure 1 displays a flow chart of the screened, excluded, and analyzed articles that were included in the final analysis. In the English-language literature search, 273 titles and abstracts were identified, and 25 full articles were retrieved for further review. Of the 25 studies, three did not include pain outcome assessment [60-62], one was a review article [52], two had no control comparison $[63,64]$, and four included cointerventions, including acupuncture $[65,66]$, mobilization [28], and Internet information [34]. Thus, only 15 English-language studies were included for further analysis. In the Chinese-language literature search, 179 titles and abstracts were identified, and nine full articles were retrieved and reviewed. Of the nine studies, one study was a review paper [67], one study did not have a sham group [68], and one included acupuncture [69]. These three studies were therefore excluded. Ultimately, we included a total of 22 (15 English and 6 Chinese) studies in our metaanalysis, which were RCTs assessing the effect(s) of auricular therapy.

3.2. Characteristics of Included Studies. Table 1 lists the characteristics of the studies included for analysis. Of the 22 RCTs included, seven studies had scores of over 70 on methodological quality, with a mean score of 66.28 (SD = 8.84, range $=45.70-79.00$ ) for English-language studies and 54.83 ( $\mathrm{SD}=9.66$, range $=40.50-70.00)$ for Chineselanguage studies. The mean scores of English-language studies were significantly higher than Chinese-language studies $(P=0.024)$. One English-language study [26] and one Chinese language study [38] scored less than 50, which indicates a lower MS. The countries where the studies were conducted included Europe $(n=9)$, the United States $(n=5)$, China $(n=6)$, and Taiwan $(n=2)$. Studies conducted in the United States took place mainly in military settings $(n=3)$. The sample size ranged from 19 to 180 , with a mean of 62 . Studies conducted in China or Taiwan tended to have larger sample size $(n \geq 60)$.

A VAS scale (0-10 or 0-100) was used for all pain outcome measures. Among the 22 trials, auricular therapy methods included AA $(n=10)$, EAS $(n=4)$, and AP $(n=8)$. The type of pain included perioperative pain $(n=9)$, acute pain $(n=7)$, and chronic pain $(n=6)$. The treatment duration ranged from one treatment (AA) to weekly AA treatment for up to 6 weeks [24]. The most popular acupoints selected for treatment were corresponding points ( $n=20)$, shenmen $(n=17)$, and subcortex (also called dermis) $(n=8)$. Seven trials included a sham control (using sham acupoints) for comparison of auricular effects in pain relief, and the selection of sham acupoints was based on using points outside the pain zone area on the ear. An electrical point finder was used in most cases to find the acupoints for treatment $(n=8)$. Bilateral acupoints were used for treatment in six trials, and unilateral acupoints were used in four trials. Twelve trials did not specify whether bilateral or unilateral acupoints were used. Most of the trials reported positive outcomes; however, one trial showed AA was less effective when compared to local analgesic use [27]; two trials reported mixed results (multiple times points of pain scores) $[13,23]$.

3.3. Meta-Analysis (Effects of Intervention). In the final metaanalysis, nine studies were excluded because we were not able to retrieve raw data (i.e., mean and standard deviation), which included five English-language studies [22, 23, 25, 30, 70] and four Chinese-language studies [37-40]. Due to the different methods of treatment (including AA, EAS, and AP) and great variation of study endpoints among the trials, findings in this meta-analysis were presented according to different treatment methods (follow-up duration, which included immediate (within 15 minutes), 12 to 24 hours after treatment, 24 to 48 hours after treatment, and long term follow-up). 


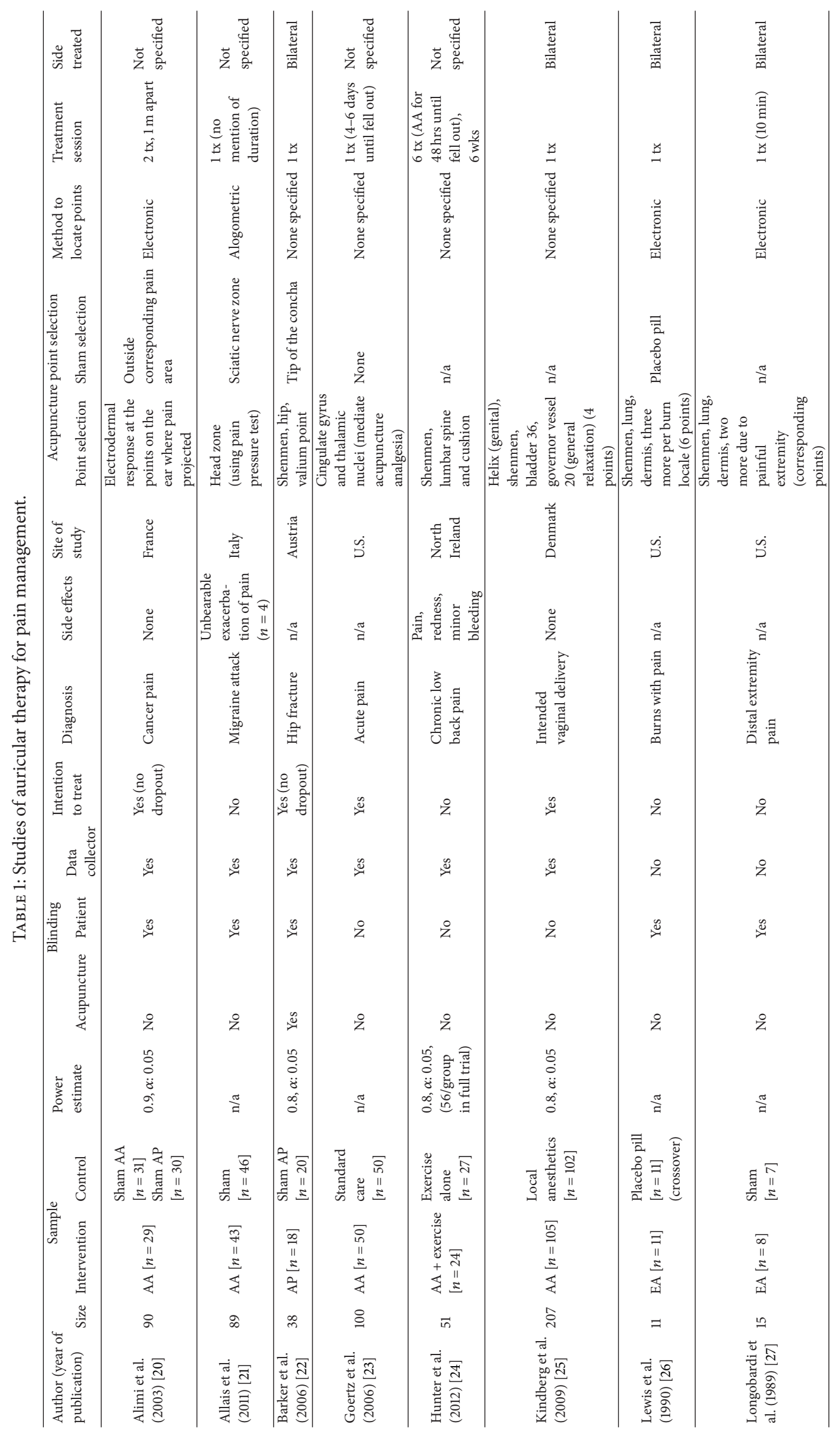




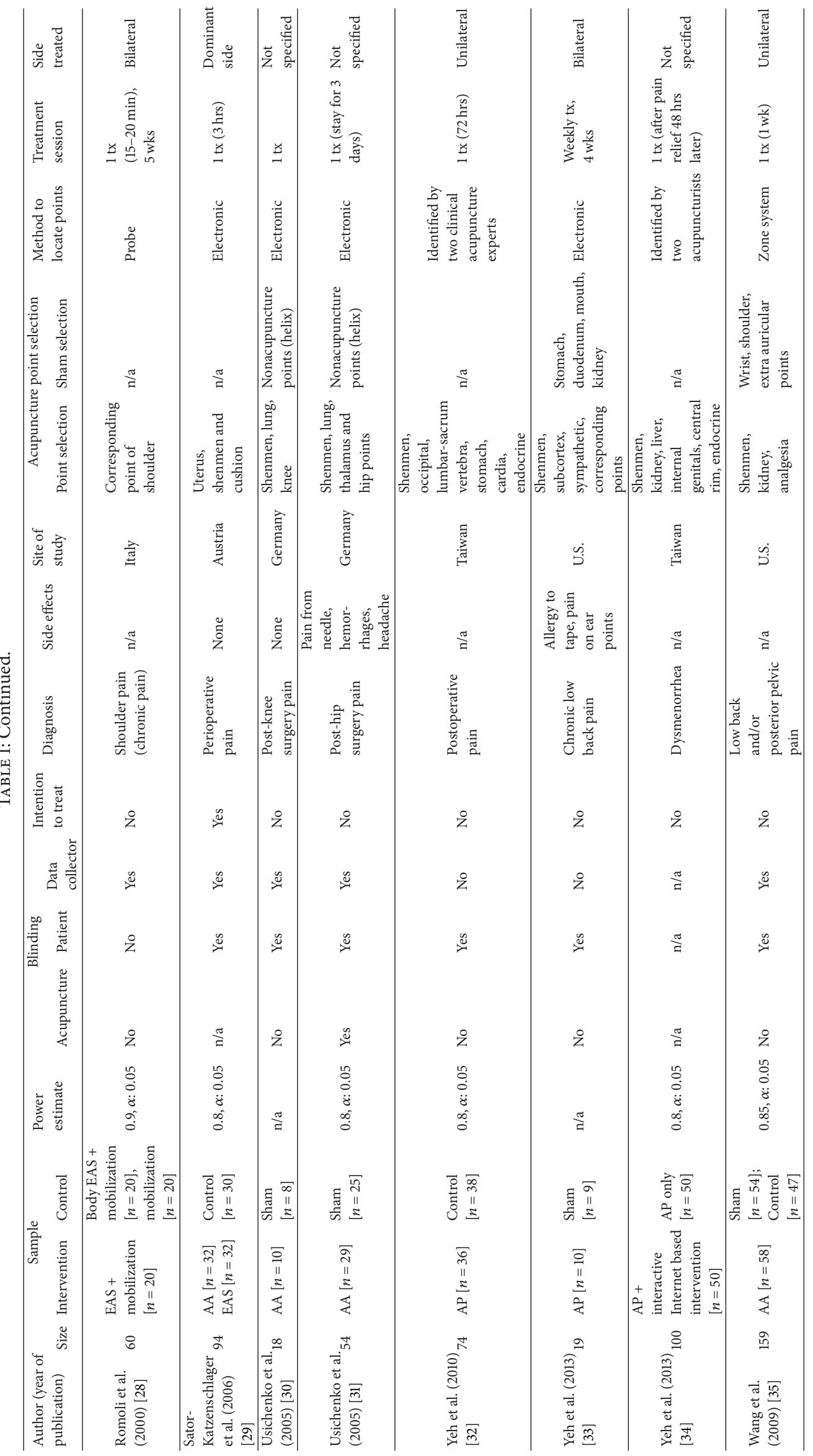




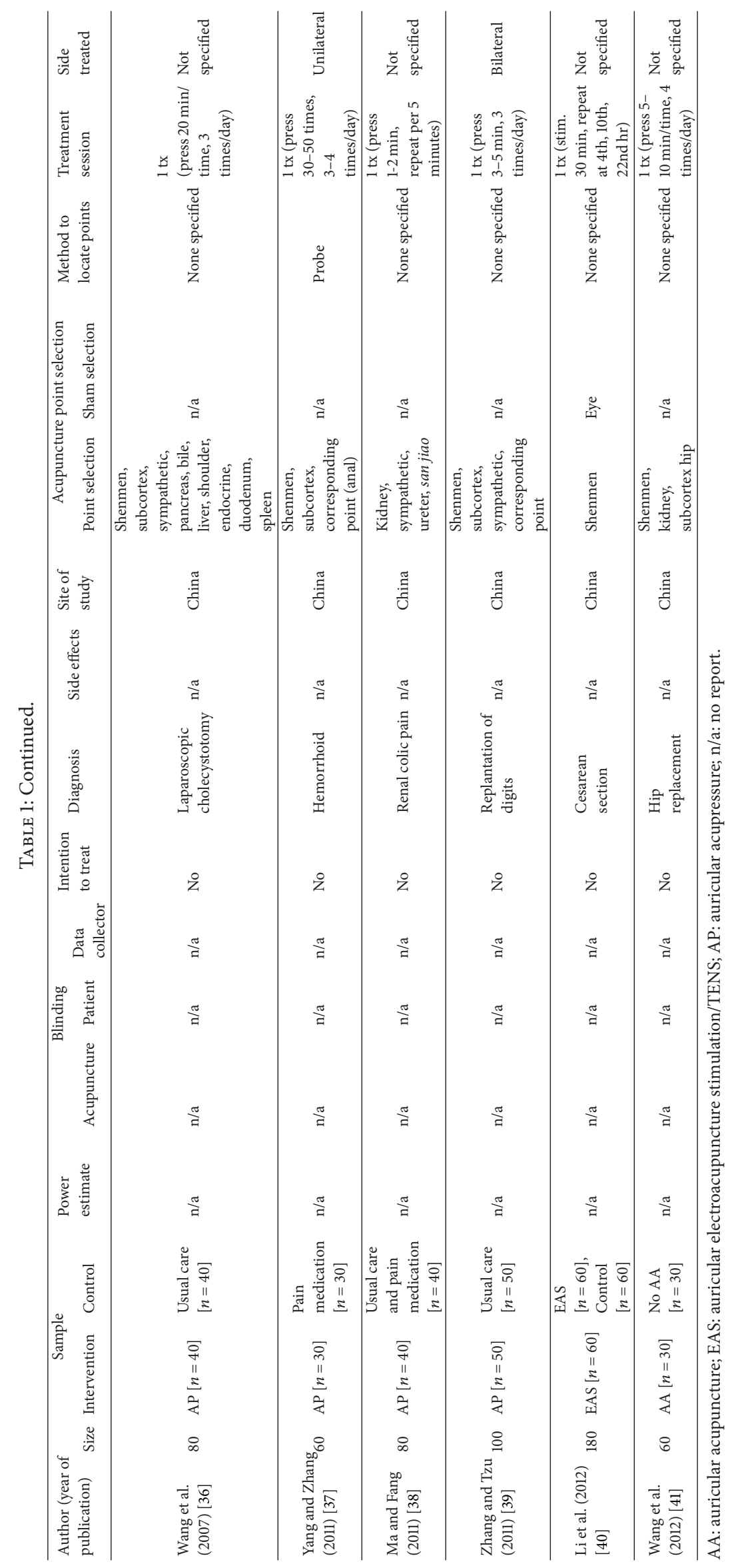




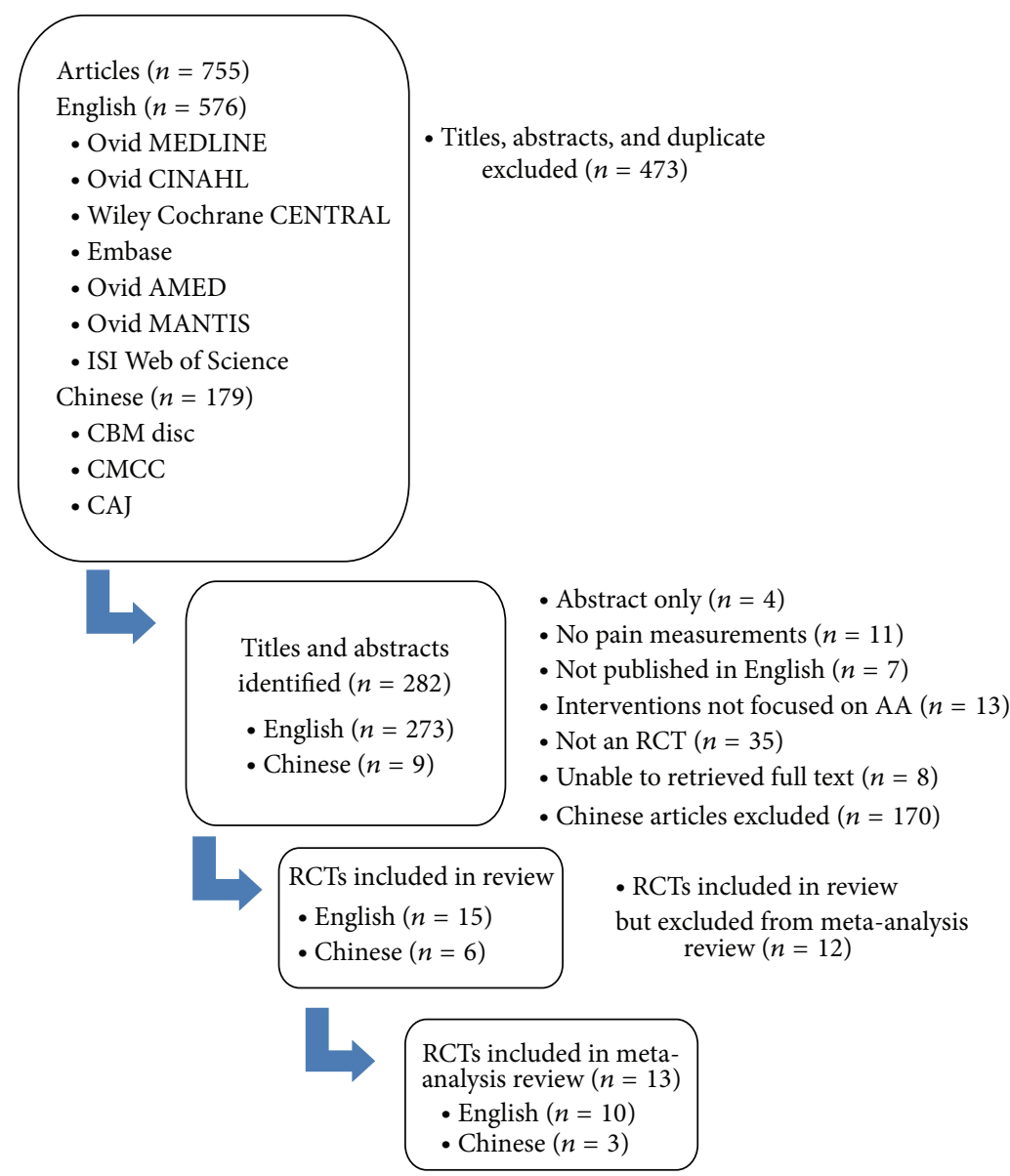

FIGURE 1: Flow chart of screened, excluded, and analyzed articles.

TABLE 2: Ovid MEDLINE search for auricular therapy for pain management article.

\section{Search string}

\section{(1) Acupuncture}

(2) Acupuncture, ear

(3) Auriculotherapy

(4) Ear, external

(5) Acupuncture therapy or acupressure

(6) 4 and 5

(7) Auriculotherapy.ti,ab.

(8) Auricular therapy.ti,ab.

(9) Aural therapy.ti,ab.

(10) Ear acupuncture.ti,ab.

(11) Ear acupressure.ti,ab.

(12) Auricular acupuncture.ti,ab.

(13) Auricular acupressure.ti,ab.

(14) 1 or 2 or 5 or 6 or 7 or 8 or 9 or 10 or 11 or 12

(15) Limit 13 to "therapy (sensitivity)"

3.4. Overall Pain Relief of Auricular Therapy for 13 Studies. Figure 2 presents the findings of the 13 trials included for meta-analysis. Among these 13 trials, two studies [20, 43] used a 0-100 scale to measure pain, while the eleven other studies used a 0-10 scale. Seven studies reported statistically significant pain relief of auricular therapy compared to the sham group $[20,21,32,34,36,39,41,43]$, while six studies found no significant difference in pain relief between auricular therapy and the sham control [24, 26, 27, 29, 31, 32]. Among the 13 trials, auricular therapy was found to be a significant method of pain relief when compared to the sham or control group $(\mathrm{SMD}=-1.59,95 \%$ CI $[-2.36,-0.82], P=$ $0.001)$. Highly significant heterogeneity was found among the 13 studies $\left(\chi^{2}=262.30, I^{2}=95 \%, P<0.01\right)$, indicating their heterogeneity. We conducted further sensitivity testing and removed two studies that showed much larger effect than the other studies [21, 35]. In doing this, heterogeneity was reduced $\left(\chi^{2}=51.23, I^{2}=80 \%, P<0.01\right)$ and the SMD decreased to 0.69 (95\% CI $[-1.08,-0.30])$. The overall strength of the evidence for the efficacy of auricular therapy for pain relief was rated as medium to large.

3.5. Pain Relief vis-à-vis Different Auricular Therapy Treatment Methods. Among the seven studies featuring AA, AA was found to be a significant method of pain relief when compared to the sham or control group (SMD = $-1.81,95 \%$ CI $[-2.92,-0.70], P=0.001$ ) (Figure 3 ). Highly 
TABLE 3: Methodological quality (developed by Ter Riet) (score 0-100).

Study population (35 points)

(A) Homogeneity (2)

(B) Comparability of prognoses at baseline (5)

(C) Adequate randomization procedure (4)

(D) Dropouts described for each treatment group separately (4)

(E) Loss to follow-up (8)

(F) Study size (12)

Intervention (25 points)

(G) Interventions included in protocol and described (10)

(H) Pragmatic study (5)

(I) Cointerventions avoided (or comparable) (5)

(J) Placebo-controlled (5)

Measurement of effect (30 points)

(K) Patient blinded (5)

(L) Outcome measures relevant (10)

(M) Validity/reliability of instruments (5)

(N) Blinded outcomes assessments (5)

(O) Follow-up period adequate (5)

Data presentation and analysis (10 points)

(P) Intention-to-treat analysis (5)

(Q) Data presented for most important outcome measures (5)

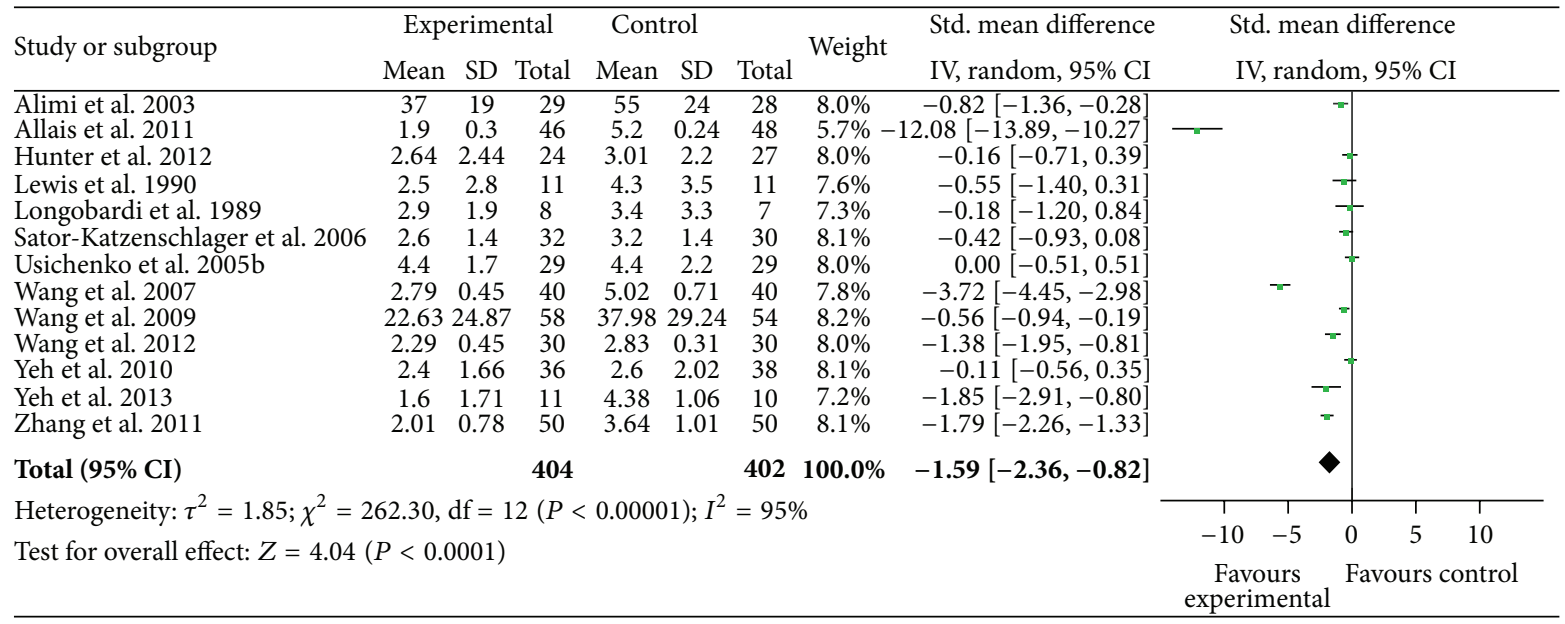

Figure 2: Pain relief of auricular therapy for 13 trials.

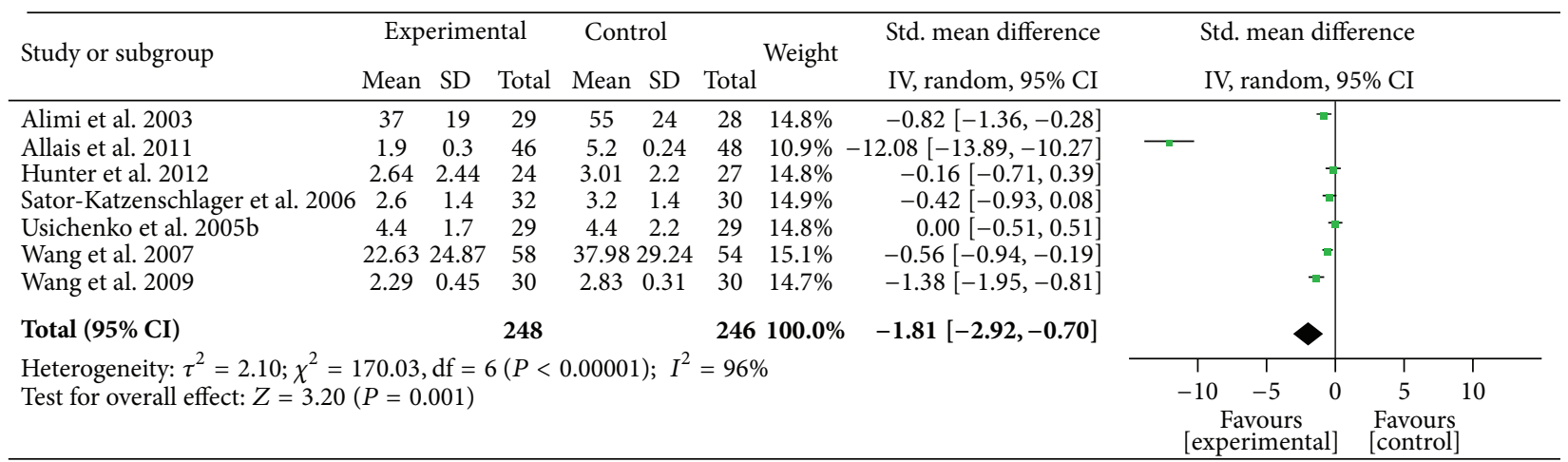

FIGURE 3: Pain relief of auricular therapy using auricular acupuncture. 


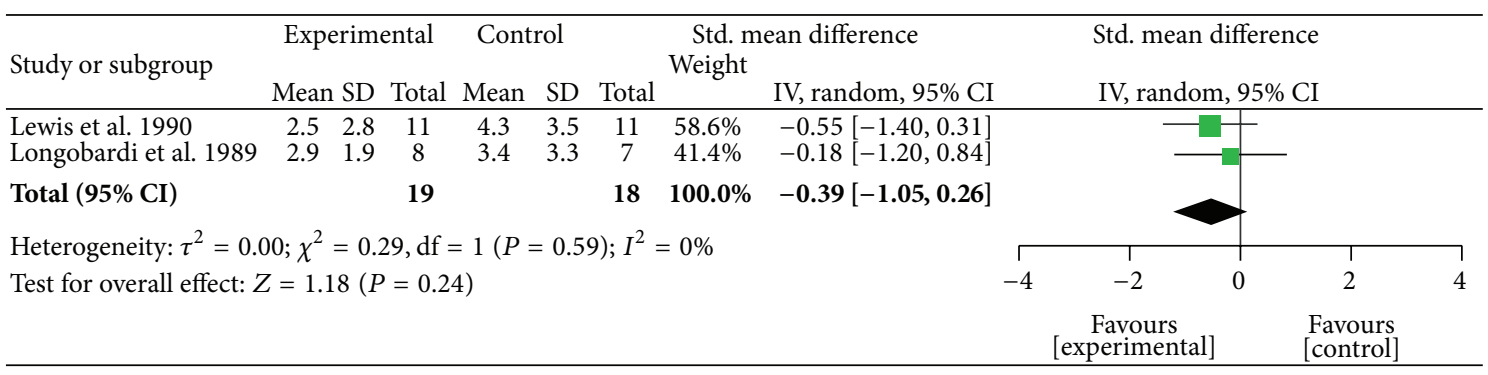

FIGURE 4: Pain relief of auricular therapy using electroacupuncture stimulation.

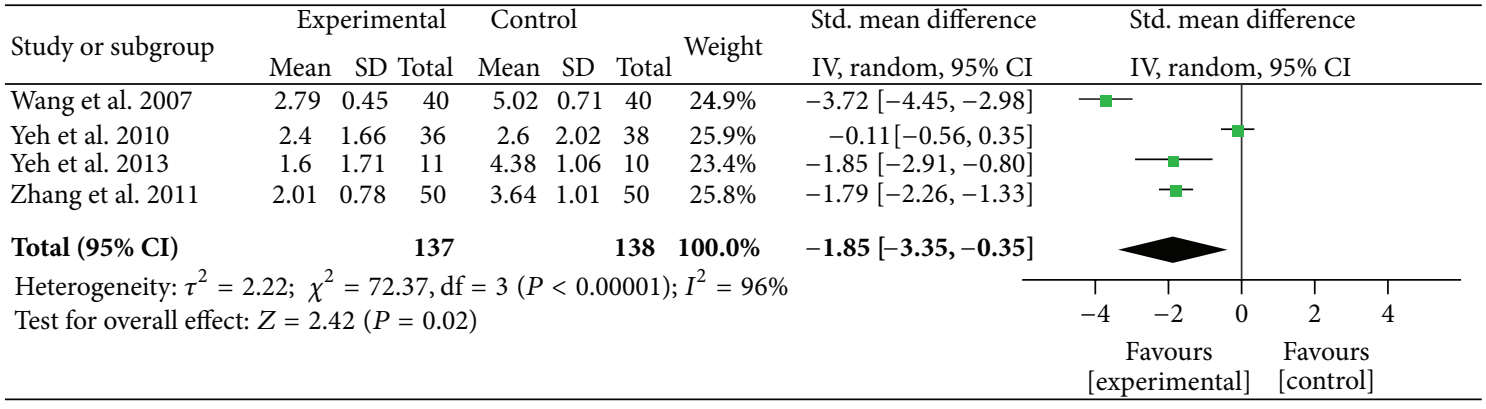

FIGURE 5: Pain relief of auricular therapy using auricular acupressure.

significant heterogeneity was found among the studies $\left(\chi^{2}=\right.$ $\left.170.03, I^{2}=96 \%, P<0.001\right)$, indicating their heterogeneity. Publication bias was assessed by funnel plot and asymmetry was observed, which suggested potential publication bias due to the study by Allais et al. [21]. We conducted further sensitivity testing and removed the Allais et al. study. After removal of the study [21], AA was found to be significant for pain relief when compared to the sham or control group $(\mathrm{SMD}=-0.55,95 \% \mathrm{CI}[-0.91,-0.19], P=0.003, n=$ 202). The overall strength of the evidence for the efficacy of auricular therapy for pain relief was rated as medium to large. Among the two studies using EAS, EAS was found to be nonsignificant for pain reduction when compared to the sham or control group (SMD $=-0.39 ; 95 \%$ CI $[-1.05,0.26]$; $P=0.24, n=19$ ) (Figure 4). Among the four studies using AP, AP was found to be a significant method for pain relief when compared to the sham or control group $(\mathrm{SMD}=-1.85$, $95 \%$ CI $[-3.35,-0.35], P=0.002$ ) (Figure 5). The overall strength of the evidence for the efficacy of auricular therapy for pain relief was rated as large.

3.6. Immediate Pain Relief after Auricular Therapy (within 15 Minutes after Treatment). Four studies compared the immediate pain relief of auricular therapy (15 minutes or less) for treating migraine using AA [21], pain with burns using EAS [26], perioperative pain during oocyte aspiration in IVF treatment using EAS [29], and distal extremity pain using EAS [27]. Heterogeneity tests were significant $\left(\chi^{2}=146.98\right.$, $\left.I^{2}=98 \%, P<0.01\right)$, which indicates statistical evidence for differences between the four studies (Figure 6). Intervention groups tended to have lower scores of pain intensity than sham groups; however, only one study reached statistical significance [21]. The combined mean difference for the four studies showed nonsignificant pain reduction for immediate effect measures (SMD $=-2.84 ; 95 \%$ CI $[-5.92,0.24] ; P=$ 0.07, $n=193)$.

3.7. Pain Relief after Auricular Therapy (12 to 24 Hours after Treatment). Four studies included pain intensity measured at 12 to 24 hours after auricular therapy. The four studies showed significant heterogeneity $\left(\chi^{2}=144.59, P<0.001\right)$ (Figure 7$)$. Two studies had significant pain relief at 12 to 24 hours after auricular therapy $[36,39]$. The combined mean difference for these four studies did not reach statistical significance $(\mathrm{SMD}=-1.71 ; 95 \% \mathrm{CI}[-3.67,0.24] ; P=0.09 ; n=314)$ (Figure 8).

3.8. Pain Relief after Auricular Therapy (24 to 48 Hours after Treatment). Four studies examined pain relief at 24 to 48 hours after auricular therapy and displayed good quality [31, 32, 39, 41]. Heterogeneity between studies was highly significant $\left(\chi^{2}=89.05, P<0.001\right.$ ) (Figure 8 ). Two studies demonstrated statistically significant pain relief after auricular therapy at 24 to 48 hours $[39,41]$, while the other two trials did not show pain relief $[31,39]$. Among the four trials, auricular therapy did not show significant pain relief after 24 to 48 hours when compared to sham groups (SMD = -1.39 ; 95\% CI $[-2.84,0.05]$; $P=0.006 ; n=306)$.

\section{Discussion}

In this meta-analysis, we had the advantage of including both English- and Chinese-language studies, which was not done in Asher et al.'s previous meta-analysis [52]. The main finding 


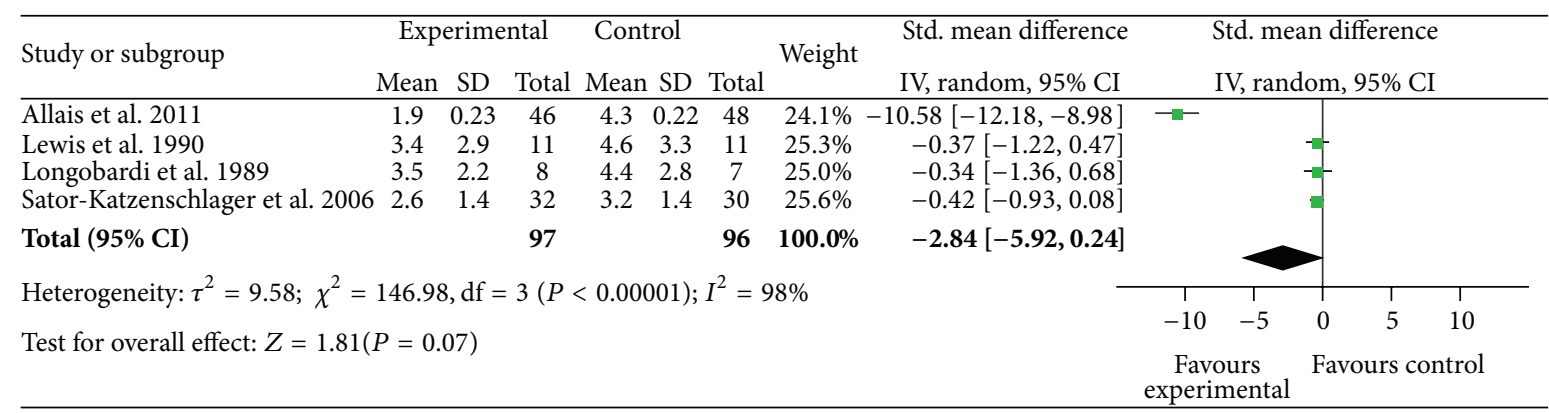

FIGURE 6: Immediate pain relief after auricular therapy (within 15 minutes).

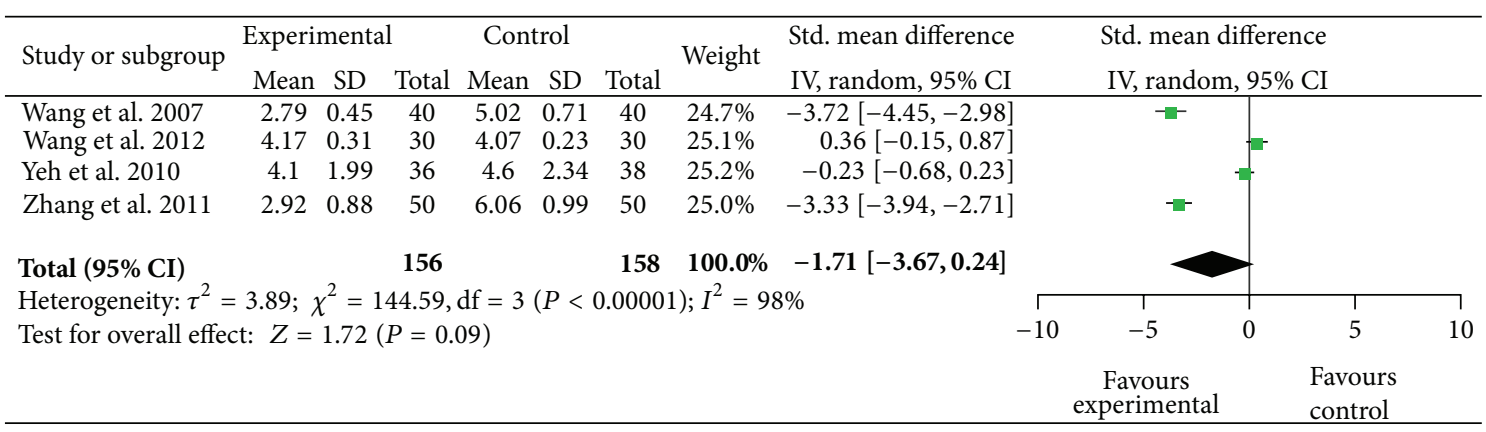

FIgURE 7: Immediate pain relief after auricular therapy (12 to 24 hours after treatment).

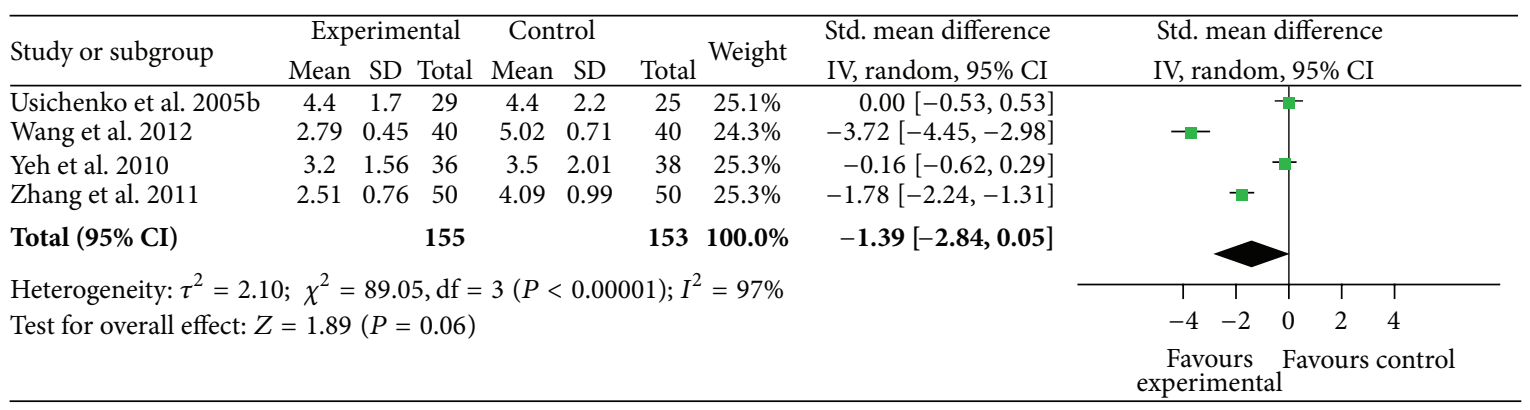

FIGURE 8: Pain relief after auricular therapy (24 to 48 hours after treatment).

of this study is that auricular therapy provided significant pain relief when compared to sham or control groups in the various studies analyzed. The overall SMD was 1.59 $(95 \%$ CI $[-2.36,-0.82])$ (13 trials, total subject number $=$ $806)$, indicating that, on average, the mean decrease in pain score for auricular therapy group was 1.59 standard deviations greater than the mean decrease for the sham control, which is similar to the earlier meta-analysis (SMD = $1.56,95 \%$ CI $[0.82,2.26])$ (8 trials, $n=387$ ) [52]. Findings from that meta-analysis and our meta-analysis demonstrate significant heterogeneity of the studies included. When attempts were made to remove particular individual studies, heterogeneity was reduced but still existed, and SMD dropped to $-0.69\left(I^{2}=80 \%, \mathrm{CI}:-1.08,-0.30\right)$ for this study and $1.01\left(I^{2}=74 \%, 95 \%\right.$ CI: $\left.0.51,1.51\right)$ for Asher et al.s study [52]. Compared to Asher et al., which included six studies $(n=303)$ in the meta-analysis, our meta-analysis had 13 studies with larger sample size $(n=806)$, which leads to a smaller effect size. Hence, our study more accurately represents the efficacy of auricular therapy for pain relief, with moderate- to high-strength evidence.

Before interpreting the findings of this study, we need to be aware of its limitations. First, the results of this metaanalysis depend on the quality of the studies included. The quality of the studies included had MQ mean scores of 65.82 for English-language studies and 54.83 for Chinese-language studies. According to the definition of MQ, a score of 50 indicates good quality. In the studies included in this metaanalysis, 91\% were rated as good, which is much higher than the previous meta-analysis which had only $35 \%$ rated as good [52]. Moreover, the differences in study parameters are likely responsible for the varying treatment effect due to 
significant variation, including acupoint selection, form of auricular therapy, treatment duration, and study endpointsall of which could impact the quality of the studies. Second, we only included published studies, which may skew the results because most studies with negative results were unpublished. Finally, only seven studies (32\%) used a sham group for comparison. Without the sham comparison, the effect of auricular therapy for pain is subject to placebo effects, patient expectations on treatment outcome, and given patient's relationship with his or her therapist.

Acupoint selection for both treatment and sham control groups is a critical factor impacting treatment effects. Differing from body acupuncture, which works on the meridian basis (i.e., health is promoted through the balancing of $y \mathrm{in}$ and yang), auricular therapy works on a microsystem basis, with the ear as a self-contained microsystem that can affect the state of the entire body. Based on Nogier's theory [71], somatotopic correspondences can be found between auricular points and their corresponding (i.e., projected) body areas. When a person suffers from disease in a particular part of the body, the auricular acupoints may show not only a decrease in auricular cutaneous electrical resistance, but also a decrease in pain threshold [72]. Thus, corresponding acupoints are the key acupoints for treatment in auricular therapy. In this metaanalysis, corresponding acupoints $(91 \%, n=20)$ and shenmen (a general analgesic point) $(77 \%, n=17)$ are the most commonly used acupoints for pain treatment. The positive outcomes for the majority of studies included in this metaanalysis suggest that auricular acupoints are indeed specific to particular diseases or symptoms. However, we need to be cautious about this conclusion because only $32 \%(n=7)$ of the total studies included in our meta-analysis had a sham group comparison.

In the studies that included a sham group comparison, the selection of sham acupoints consisted of simply placing them outside the corresponding pain area, which is reasonable considering the somatotopic mapping between auricular acupoints and body. However, none of these studies acknowledged that the selection of these sham acupoints may conflict with TCM meridian and channel theory. In other words, what is intended to be a sham acupoint in terms of one auricular paradigm could actually influence an intervention's outcomes, positively or negatively, according to another auricular paradigm.

Auricular therapy, a form of acupuncture, has a history of more than 2,000 years in TCM. Therefore, it still shares some key treatment parameters of TCM meridian and channel theory that were followed by the researchers of the studies assessed in this meta-analysis. For example, some auricular acupoints are one-point, multiple-disease, according to TCM zang-fun theory. In one study, the lung acupoint was used to reduce pain during incisions [27], based on the association between lung and the body skin surface in TCM theory. However, to date, there have been no empirical studies that discuss the treatment outcomes of acupoint selection by comparing the projection of somatic topography with TCM meridian and channel theory. In addition, although auricular acupoints are basically the same between the Chinese and Nogier systems, the ear charts do vary in somatotopic arrangement, particularly with regard to spinal, internal organ, and reproductive organ location [17]. Therefore, more empirical evidence is needed to establish the validity of ear charts between the Chinese and Nogier approaches to auricular therapy.

Treatment duration (i.e., how long the treatments provide benefit) is another key factor for treatment outcomesparticularly for chronic pain. In this meta-analysis, the treatment duration for chronic pain varied from one-time treatment to up to six treatments. The lack of agreement on the optimal duration of auricular therapy may mean that some patients do not receive the best treatment. In general, 2 to 10 weeks of auricular therapy has been reported to provide treatment benefits $[17,19]$; however, these reports lack empirical evidence. In acupuncture, six treatments over a 3-week period provide better pain relief than does the administration of fewer than six treatments [73]. Studies on the sustained effects of auricular therapy for pain relief are varied and report findings ranging from immediately [21] to up to 6 months [24], but the findings are limited by small sample size [52]. In a study of AP for chronic low back pain, 1 week of AP can reduce pain intensity by $45 \%$ [74], and 4 weeks of APA can achieve even more pain relief (i.e., 75\% reduction) and maintain such effects for 1 month [33]. Studies have established evidence of auricular therapy, yet more empirical studies are needed to establish an adequate assessment of treatment duration.

In terms of the efficacy of the different treatment methods, AP boasts the largest strength of evidence for the efficacy for pain relief, followed by AA. EAS did not show significant evidence for efficacy, which may be due to the small sample size (i.e., only 19 subjects were included). Compared to AP, AA and EAS are more popular in Europe and the United States. Additionally, these techniques usually need to be performed by qualified physicians. In contrast, studies conducted in China and Taiwan primarily feature AP, and $\mathrm{AP}$ is a noninvasive, low-cost, and self-managed approach for patients. The application of AP utilizes botanical plant seeds or magnetic pellets taped on the both sides of the ears to stimulate acupoints. Once seeds are applied with tape by a trained therapist, the taped-on seeds can remain on the ears for 1 to 3 weeks, depending on the skin condition of the ear. The biggest advantage of AP is that patients themselves can stimulate the acupoints by pressing them with the thumb and forefinger as directed to achieve acupuncture-like effects. Patients are instructed to press the acupoints whenever they want to decrease pain. With this patient involvement, fewer visits to a therapist are required for auricular acupressure compared to body acupuncture. Although auricular therapy (especially AP) can be easily administered by a trained practitioner, further research should investigate whether or not it could be self-administered and whether or not accurate seed placement could be achieved by the patient.

A definitive elucidation of the underlying biological mechanism of auricular therapy in treating pain remains elusive. One theoretical explanation of auricular therapy is that pain and neuronal excitability are relieved by normalizing pathological, hypersensitive reflex pathways (i.e., the neural immune pathway) that interconnect the ear microsystem and 
the somatotopic region of the brain $[17,19]$. The neurophysiological connections between ear acupoints and the human CNS have been corroborated by fMRI [75]. The stimulation of acupoints is thought to cause vasodilative effects by releasing either beta-endorphin to elicit short-term analgesic effects or neuropeptide-induced anti-inflammatory cytokines for longterm effects [76-78]. Considering the complex interaction between cytokines, neuropeptides, and neurotrophins pertaining to chronic pain, possible pathways of the ameliorating effect of auricular therapy on pain include (1) the downregulation of proinflammatory cytokines and the upregulation of anti-inflammatory cytokines, (2) the downregulation of proinflammatory neuropeptides (e.g., calcitonin gene-related peptide), and (3) the downregulation of neurotrophins (e.g., nerve growth factor, NGF) $[77,79]$. These responses may be modulated by inflammatory mediators and could explain the analgesic effects of auricular therapy.

\section{Conclusion}

Our findings suggest that auricular therapy can be used as an adjunct therapy for pain management and, therefore, reduce analgesic use to minimize potential adverse effects and tolerance. Nonetheless, further studies-particularly large scale of RCTs-are needed to further confirm the efficacy of auricular therapy for pain and must take into consideration important features of methodological design, which include point specification, stimulation, treatment duration, placebo effects, and patient expectations of treatment outcomes.

\section{Conflict of Interests}

The authors declare that there is no conflict of interests regarding the publication of this paper.

\section{Acknowledgments}

This study was supported by a grant awarded to Dr. Yeh from the Center for Research and Evaluation (CRE) Pilot/Feasibility Study Program, School of Nursing, University of Pittsburgh, and the Aging Institute of the University of Pittsburgh Medical Center (UPMC), Senior Services. The authors also wish to thank Brian Greene in the CRE, who edited several drafts of this paper prior to submission.

\section{References}

[1] WHO Scientific Group, "The burden of musculoskeletal conditions at the start of the new millennium," World Health Organization Technical Report Series, vol. 919, no. 1-10, pp. 1$218,2003$.

[2] D. Hoy, P. Brooks, F. Blyth, and R. Buchbinder, "The Epidemiology of low back pain," Best Practice and Research: Clinical Rheumatology, vol. 24, no. 6, pp. 769-781, 2010.

[3] CDC, "Prevalence of disabilities and associated health conditions among adults-United States," $M M W R$, vol. 58, pp. 421426, 2009.
[4] L. Vorvick, C. Ma, and D. Zieve, "Low back pain-acute," U.S. National Library of Medicine, 2012, http://www.ncbi.nlm.nih .gov/pubmedhealth/PMH0004668/.

[5] J. L. St. Sauver, D. O. Warner, B. P. Yawn et al., "Why patients visit their doctors: assessing the most prevalent conditions in a defined American population," Mayo Clinic Proceedings, vol. 88, no. 1, pp. 56-67, 2013.

[6] S. Dagenais, J. Caro, and S. Haldeman, "A systematic review of low back pain cost of illness studies in the United States and internationally," Spine Journal, vol. 8, no. 1, pp. 8-20, 2008.

[7] J. K. Freburger, G. M. Holmes, R. P. Agans et al., "The rising prevalence of chronic low back pain," Archives of Internal Medicine, vol. 169, no. 3, pp. 251-258, 2009.

[8] T. W. Strine and J. M. Hootman, "US national prevalence and correlates of low back and neck pain among adults," Arthritis Care and Research, vol. 57, no. 4, pp. 656-665, 2007.

[9] M. Du Bois, M. Szpalski, and P. Donceel, "Patients at risk for long-term sick leave because of low back pain," The Spine Journal, vol. 9, no. 5, pp. 350-359, 2009.

[10] G. Malanga and E. Wolff, "Evidence-informed management of chronic low back pain with nonsteroidal anti-inflammatory drugs, muscle relaxants, and simple analgesics," The Spine Journal, vol. 8, no. 1, pp. 173-184, 2008.

[11] R. Benyamin, A. M. Trescot, S. Datta et al., "Opioid complications and side effects," Pain Physician, vol. 11, no. 2, pp. S105S120, 2008

[12] M. Maltoni, “Opioids, pain, and fear," Annals of Oncology, vol. 19, pp. 5-7, 2008.

[13] A. K. Kanodia, A. T. R. Legedza, R. B. Davis, D. M. Eisenberg, and R. S. Phillips, "Perceived benefit of Complementary and Alternative Medicine (CAM) for back pain: a national survey," Journal of the American Board of Family Medicine, vol. 23, no. 3, pp. 354-362, 2010.

[14] A. J. Vickers, A. M. Cronin, A. C. Maschino et al., "Acupuncture for chronic pain: individual patient data meta-analysis," Archives of Internal Medicine, vol. 172, no. 19, pp. 1444-1453, 2012.

[15] R. L. Nahin, P. M. Barnes, B. J. Stussman, and B. Bloom, "Costs of complementary and alternative medicine (CAM) and frequency of visits to CAM practitioners: United States, 2007," National Health Statistics Reports, no. 18, pp. 1-14, 2009.

[16] D. M. Upchurch and B. W. Rainisch, "A sociobehavioral wellness model of acupuncture use in the United States, 2007," Journal of Alternative and Complementary Medicine, vol. 20, no. 1, pp. 3239, 2013.

[17] LC. Huang Auricular International Research \& Training, Orlando Fla, USA.

[18] L. J. Spagrud, C. L. von Baeyer, K. Ali et al., "Pain, distress, and adult-child interaction during venipuncture in pediatric oncology: an examination of three types of venous access," Journal of Pain and Symptom Management, vol. 36, no. 2, pp. 173-184, 2008.

[19] T. Oleson, Auriculotherapy Manual: Chinese and Western Systems of Ear Acupuncture, Churchill Livingstone, Kidlington, UK, 2003.

[20] D. Alimi, C. Rubino, E. Pichard-Léandri, S. Fermand-Brulé, M.L. Dubreuil-Lemaire, and C. Hill, "Analgesic effect of auricular acupuncture for cancer pain: a randomized, blinded, controlled trial," Journal of Clinical Oncology, vol. 21, no. 22, pp. 4120-4126, 2003. 
[21] G. Allais, M. Romoli, S. Rolando et al., "Ear acupuncture in the treatment of migraine attacks: A randomized trial on the efficacy of appropriate versus inappropriate acupoints," Neurological Sciences, vol. 32, pp. 173-175, 2011.

[22] R. Barker, A. Kober, K. Hoerauf et al., "Out-of-hospital auricular acupressure in elder patients with hip fracture: a randomized double-blinded trial," Academic Emergency Medicine, vol. 13, no. 1, pp. 19-23, 2006.

[23] C. M. H. Goertz, R. Niemtzow, S. M. Burns, M. J. Fritts, C. C. Crawford, and W. B. Jonas, "Auricular acupuncture in the treatment of acute pain syndromes: a pilot study," Military Medicine, vol. 171, no. 10, pp. 1010-1014, 2006.

[24] R. F. Hunter, S. M. McDonough, I. Bradbury et al., "Exercise and auricular acupuncture for chronic low-back pain: a feasibility randomized-controlled trial," The Clinical Journal of Pain, vol. 28, no. 3, pp. 259-267, 2012.

[25] S. Kindberg, L. Klünder, J. Strøm, and T. B. Henriksen, "Ear acupuncture or local anaesthetics as pain relief during postpartum surgical repair: a randomised controlled trial," BJOG, vol. 116, no. 4, pp. 569-576, 2009.

[26] S. M. Lewis, J. A. Clelland, C. J. Knowles, J. R. Jackson, and A. R. Dimick, "Effects of auricular acupuncture-like transcutaneous electric nerve stimulation on pain levels following wound care in patients with burns: a pilot study," Journal of Burn Care \& Rehabilitation, vol. 11, no. 4, pp. 322-329, 1990.

[27] A. G. Longobardi, J. A. Clelland, C. J. Knowles, and J. R. Jackson, "Effects of auricular transcutaneous electrical nerve stimulation on distal extremity pain: a pilot study," Physical Therapy, vol. 69, no. 1, pp. 10-17, 1989.

[28] M. Romoli, D. van der Windt, P. Giovanzana et al., "International research project to devise a protocol to test the effectiveness of acupuncture on painful shoulder," Journal of Alternative and Complementary Medicine, vol. 6, no. 3, pp. 281287,2000

[29] S. M. Sator-Katzenschlager, M. M. Wölfler, S. A. Kozek-Langenecker et al., "Auricular electro-acupuncture as an additional perioperative analgesic method during oocyte aspiration in IVF treatment," Human Reproduction, vol. 21, no. 8, pp. 2114-2120, 2006.

[30] T. I. Usichenko, M. Hermsen, T. Witstruck et al., "Auricular acupuncture for pain relief after ambulatory knee arthroscopy-a pilot study," Evidence-based Complementary and Alternative Medicine, vol. 2, no. 2, pp. 185-189, 2005.

[31] T. I. Usichenko, M. Dinse, M. Hermsen, T. Witstruck, D. Pavlovic, and C. Lehmann, "Auricular acupuncture for pain relief after total hip arthroplasty - a randomized controlled study," Pain, vol. 114, no. 3, pp. 320-327, 2005.

[32] M. L. Yeh, M. Y. Tsou, B. Y. Lee, H. H. Chen, and Y. C. Chung, "Effects of auricular acupressure on pain reduction in patient-controlled analgesia after lumbar spine surgery," Acta Anaesthesiologica Taiwanica, vol. 48, pp. 80-86, 2010.

[33] C. H. Yeh, L. C. Chien, D. Balaban et al., "A randomized clinical trial of auricular point acupressure for chronic low back pain: a feasibility study," Evidence-based Complementary and Alternative Medicine, vol. 2013, Article ID 196978, 9 pages, 2013.

[34] M. Yeh, Y. Hung, H. Chen, J. Lin, and Y. Wang, "Auricular acupressure combined with an internet-based intervention or alone for primary dysmenorrhea: a control study," Evidencebased Complementary and Alternative Medicine, vol. 2013, Article ID 316212, 8 pages, 2013.

[35] S.-M. Wang, P. DeZinno, E. C. Lin et al., "Auricular acupuncture as a treatment for pregnant women who have low back and posterior pelvic pain: a pilot study," The American Journal of Obstetrics and Gynecology, vol. 201, no. 3, pp. 271.el-279.el, 2009.

[36] X. Q. Wang, T. S. Ye, E. R. Chen, and X. C. Dong, "Effect of perioperative strapping and pressing on the ear acupoint for pain management after laparoscopic cholecystectomy," Nursing Journal of Chinese People's Liberation Army, vol. 24, pp. 14-16, 2007 (Chinese).

[37] C. Y. Yang and C. L. Zhang, "Auricular seed treatment to reduce pain intensity for constipation," Henan Traditional Chinese Medicine, vol. 31, pp. 504-505, 2011 (Chinese).

[38] L. C. Ma and H. C. Fang, "Auricular magnetic therapy for rental chronic pain," Journal of Nurses Training, vol. 26, pp. 934-935, 2011 (Chinese).

[39] C. Zhang and W. H. Tzu, "Auricular seed therapy for pain relief in post-operative replantation of digits: 50 cases," Zhejiang Journal of Traditional Chinese Medicine, vol. 46, pp. 650-651, 2011 (Chinese).

[40] J. Li, X. Li, M. Wang, F. Shi, and H. Yu, "Effects of transcutaneous electrical stimulation of auricular Shenmen point on postoperative nausea and vomiting and patient-controlled epidural analgesia in cesarean section," Zhonghua Yi Xue Za Zhi, vol. 92, no. 27, pp. 1892-1895, 2012 (Chinese).

[41] J. Wang, H. Bao, Y. Cai, J. Zhang, and P. Tong, "Case-control study on application of auricular acupuncture for the treatment of analgesia during perioperative period in total hip arthroplasty," Zhongguo Gu Shang, vol. 25, no. 3, pp. 220-223, 2012 (Chinese).

[42] J. G. Gilbert, "Auricular complication of acupuncture," The New Zealand Medical Journal, vol. 100, no. 819, pp. 141-142, 1987.

[43] M. Wang, M. Hsu, L. Chien, C. Kao, and C. Liu, "Effects of auricular acupressure on menstrual symptoms and nitric oxide for women with primary dysmenorrhea," Journal of Alternative and Complementary Medicine, vol. 15, no. 3, pp. 235-242, 2009.

[44] D. Lewers, J. A. Clelland, J. R. Jackson, R. E. Varner, and J. Bergman, "Transcutaneous electrical nerve stimulation in the relief of primary dysmenorrhea," Physical Therapy, vol. 69, no. 1, pp. 3-9, 1989.

[45] L. C. Hwang, T. Y. Lai, H. H. Wu, J. D. Lin, and L. L. Chen, "Exploring the effect of auricular acupressure in caring dysmenorrhea," The Journal of Health Sciences, vol. 7, pp. 236-245, 2005.

[46] J. Jiang, "Clinical study and application of auricular magnet anesthesia for the operation of the thyroid," Zhen Ci Yan Jiu, vol. 20, no. 3, pp. 4-8, 1995.

[47] H. Wu, L. Bi, P. Shen, Z. Li, and P. Zhu, "Clinical observation and mechanism study on application of auricular-pressing pill for postoperative analgesia," Journal of Traditional Chinese Medicine, vol. 17, no. 1, pp. 26-31, 1997.

[48] D. Felhendler and B. Lisander, "Pressure on acupoints decreases postoperative pain," Clinical Journal of Pain, vol. 12, pp. 326-329, 1996.

[49] L. K. P. Suen, T. K. S. Wong, J. W. Y. Chung, and V. Y. B. Yip, "Auriculotherapy on low back pain in the elderly," Complementary Therapies in Clinical Practice, vol. 13, no. 1, pp. 63-69, 2007.

[50] L. K. P. Suen, T. K. S. Wong, J. W. Y. Chung, and V. Y. B. Yip, "Auriculotherapy on low back pain in the elderly," Complementary Therapies in Clinical Practice, vol. 13, pp. 63-69, 2007.

[51] T. Bao, X. Ye, J. Skinner et al., "The analgesic effect of magnetic acupressure in cancer patients undergoing bone marrow 
aspiration and biopsy: a randomized, blinded, controlled trial," Journal of Pain and Symptom Management, vol. 41, no. 6, pp. 995-1002, 2011.

[52] G. N. Asher, D. E. Jonas, R. R. Coeytaux et al., "Auriculotherapy for pain management: a systematic review and meta-analysis of randomized controlled trials," Journal of Alternative and Complementary Medicine, vol. 16, no. 10, pp. 1097-1108, 2010.

[53] T. I. Usichenko, C. Lehmann, and E. Ernst, "Auricular acupuncture for postoperative pain control: a systematic review of randomised clinical trials," Anaesthesia, vol. 63, no. 12, pp. 13431348, 2008.

[54] G. Ter Riet, J. Kleijnen, and P. Knipschild, "Acupuncture and chronic pain: a criteria-based meta-analysis," Journal of Clinical Epidemiology, vol. 43, no. 11, pp. 1191-1199, 1990.

[55] G. Ter Riet, J. Kleijnen, and P. Knipschild, "A meta-analysis of studies into the effect of acupuncture on addiction," British Journal of General Practice, vol. 40, no. 338, pp. 379-382, 1990.

[56] J. Kleijnen, P. Knipschild, and G. Ter Riet, "Clinical trials of homoeopathy," British Medical Journal, vol. 302, no. 6772, pp. 316-323, 1991.

[57] J. Kleijnen, G. Ter Riet, and P. Knipschild, "Acupuncture and asthma: a review of controlled trials," Thorax, vol. 46, no. 11, pp. 799-802, 1991.

[58] D. Cohen, Statistical Power Analysis for the Behavioral Sciences Hillsdale, Lawrence Erlbaum Associates, Mahwah, NJ, USA, 1988.

[59] J. P. T. Higgins and S. G. Thompson, "Quantifying heterogeneity in a meta-analysis," Statistics in Medicine, vol. 21, no. 11, pp. 15391558, 2002.

[60] I. Tekeoglu, B. Adak, and M. Ercan, "Investigation into the possibilities of using ear acupressure for increasing the pain threshold during athletic training," The American Journal of Acupuncture, vol. 26, no. 1, pp. 49-52, 1998.

[61] M. Zoghi and S. Jaberzadeh, "Effects of high voltage electroauriculotherapy on experimental pain threshold," Physiotherapy, vol. 88, no. 11, pp. 658-666, 2002.

[62] B. Wetzel, D. Pavlovic, R. Kuse et al., "The effect of auricular acupuncture on fentanyl requirement during hip arthroplasty: a randomized controlled trial," The Clinical Journal of Pain, vol. 27, no. 3, pp. 262-267, 2011.

[63] H. Kager, R. Likar, H. Jabarzadeh, R. Sittl, C. Breschan, and J. Szeles, "Electrical punctual stimulation (P-STIM) with ear acupuncture following tonsillectomy, a randomised, controlled pilot study," Acute Pain, vol. 11, no. 3-4, pp. 101-106, 2009.

[64] A. Holzer, U. Leitgeb, A. Spacek, R. Wenzl, H. Herkner, and S. Kettner, "Auricular acupuncture for postoperative pain after gynecological surgery: a randomized controlled trail," Minerva Anestesiologica, vol. 77, no. 3, pp. 298-304, 2011.

[65] C. Ahn, S. Lee, J. Lee, J. P. J. Fossion, and A. Sant'Ana, “A clinical pilot study comparing traditional acupuncture to combined acupuncture for treating headache, trigeminal neuralgia and retro-auricular pain in facial palsy," JAMS Journal of Acupuncture and Meridian Studies, vol. 4, no. 1, pp. 29-43, 2011.

[66] F. Ceccherelli, P. Tortora, C. Nassimbeni, R. Casale, G. Gagliardi, and G. Giron, "The therapeutic efficacy of somatic acupuncture is not increased by auriculotherapy: a randomised, blind control study in cervical myofascial pain," Complementary Therapies in Medicine, vol. 14, no. 1, pp. 47-52, 2006.

[67] W. T. Chen, G. Q. Fu, and W. D. Shen, "Progresses of studies on acupuncture analgesia for postoperative reaction," Zhen Ci Yan Jiu, vol. 38, no. 1, pp. 83-87, 2013 (Chinese).
[68] Q. Chen, Q. Wei, and X. Zhang, "Effects of electroacupuncture on supplementary analgesia and improvement of adverse reactions induced by dolantin in oocyte retrieval," Zhongguo Zhen Jiu, vol. 32, no. 12, pp. 1113-1116, 2012.

[69] D. Yang, Z. Ma, J. Han et al., "Migraine without aura treated by comprehensive auricular acupuncture: a multicentral controlled study," Zhongguo Zhen Jiu, vol. 32, no. 11, pp. 971-974, 2012 (Chinese).

[70] R. Melzack and J. Katz, "Auriculotherapy fails to relieve chronic pain. A controlled crossover study," Journal of the American Medical Association, vol. 251, no. 8, pp. 1041-1043, 1984.

[71] P. Nogier, Handbook to Auriculotherapy, Maisonneuve, Moulins-les-Metz, France, 1968.

[72] C. H. Yeh and L. C. Huang, "Comprehensive and systematic auricular diagnosis protocol," Medical Acupuncture, vol. 25, no. 6, pp. 423-436, 2013.

[73] J. Ezzo, B. Berman, V. A. Hadhazy, A. R. Jadad, L. Lao, and B. B. Singh, "Is acupuncture effective for the treatment of chronic pain? A systematic review," Pain, vol. 86, no. 3, pp. 217-225, 2000.

[74] C.-H. Yeh, L.-C. Chien, Y.-C. Chiang, and L.-C. Huang, "Auricular point acupressure for chronic low back pain: a feasibility study for 1-week treatment," Evidence-Based Complementary and Alternative Medicine, vol. 2012, Article ID 383257, 9 pages, 2012.

[75] D. Alimi, A. Geissmann, and D. Gardeur, "Auricular acupuncture stimulation measured on functional magnetic resonance imaging," Medical Acupuncture, vol. 13, pp. 18-21, 2002.

[76] Z. Zhao, "Neural mechanism underlying acupuncture analgesia," Progress in Neurobiology, vol. 85, no. 4, pp. 355-375, 2008.

[77] F. J. Zijlstra, I. van den Berg-de Lange, F. J. P. M. Huygen, and J. Klein, "Anti-inflammatory actions of acupuncture," Mediators of Inflammation, vol. 12, no. 2, pp. 59-69, 2003.

[78] H. Kim, D. Roh, S. Yoon et al., "The anti-inflammatory effects of low- and high-frequency electroacupuncture are mediated by peripheral opioids in a mouse air pouch inflammation model," Journal of Alternative and Complementary Medicine, vol. 12, no. 1, pp. 39-44, 2006.

[79] J. Wilkinson and R. Faleiro, "Acupuncture in pain management," Continuing Education in Anaesthesia, Critical Care \& Pain, vol. 7, no. 4, pp. 135-138, 2007. 


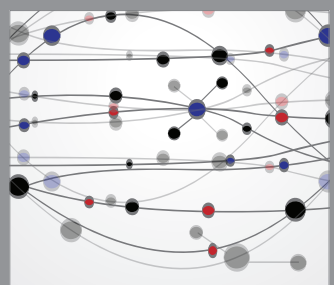

The Scientific World Journal
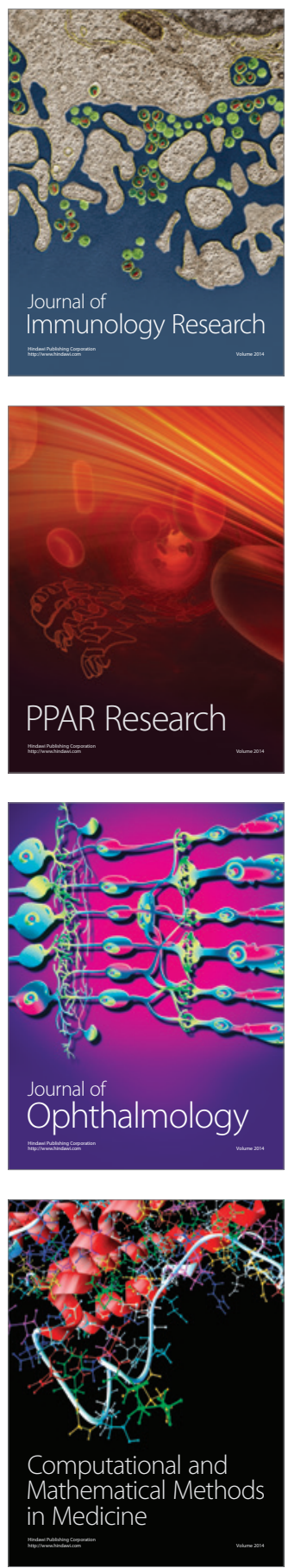

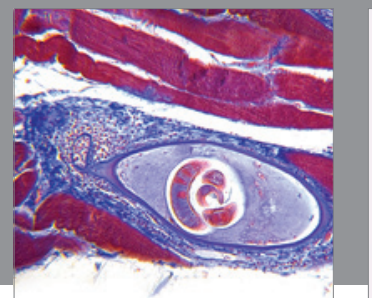

Gastroenterology

Research and Practice
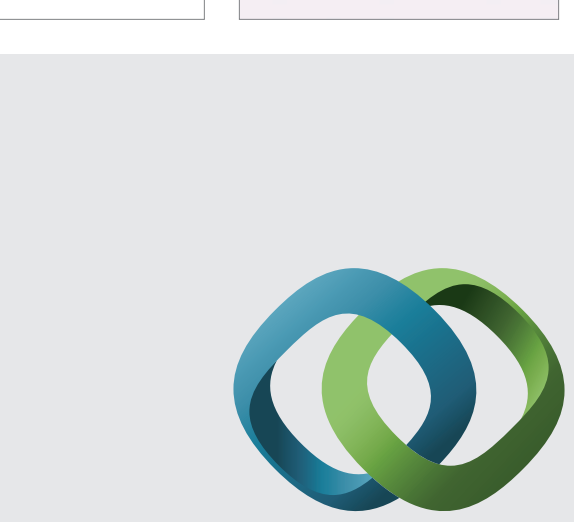

\section{Hindawi}

Submit your manuscripts at

http://www.hindawi.com
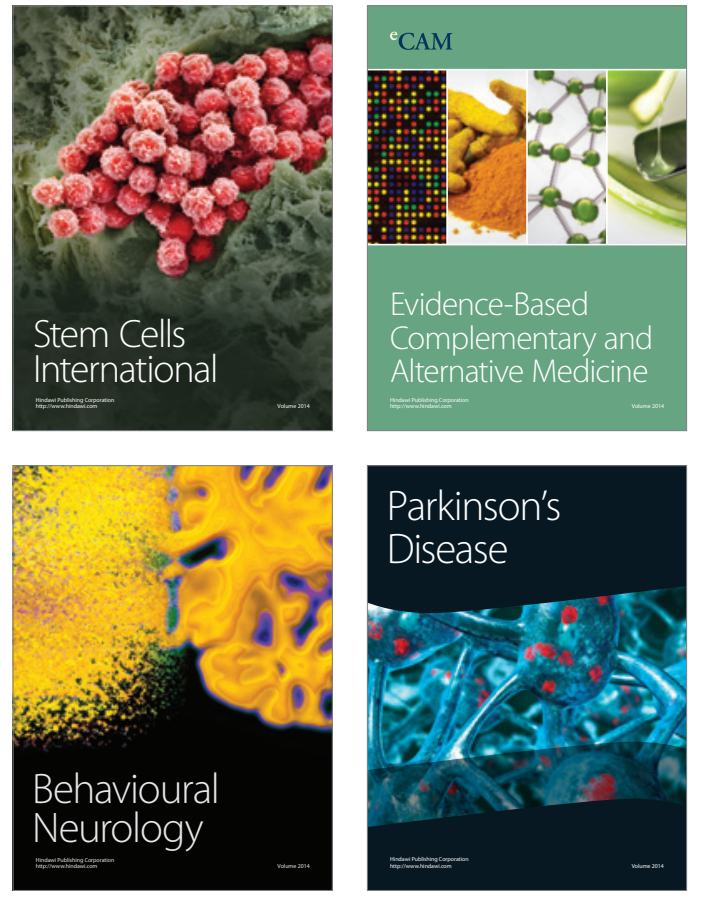
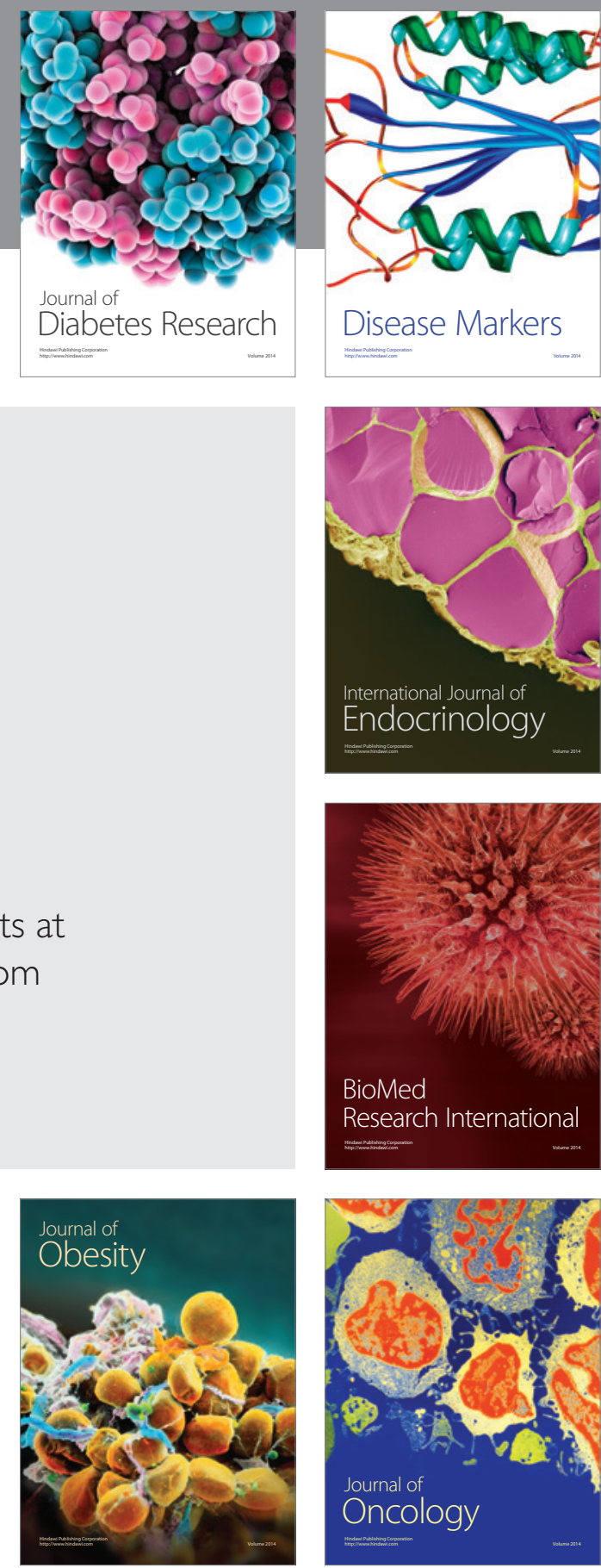

Disease Markers
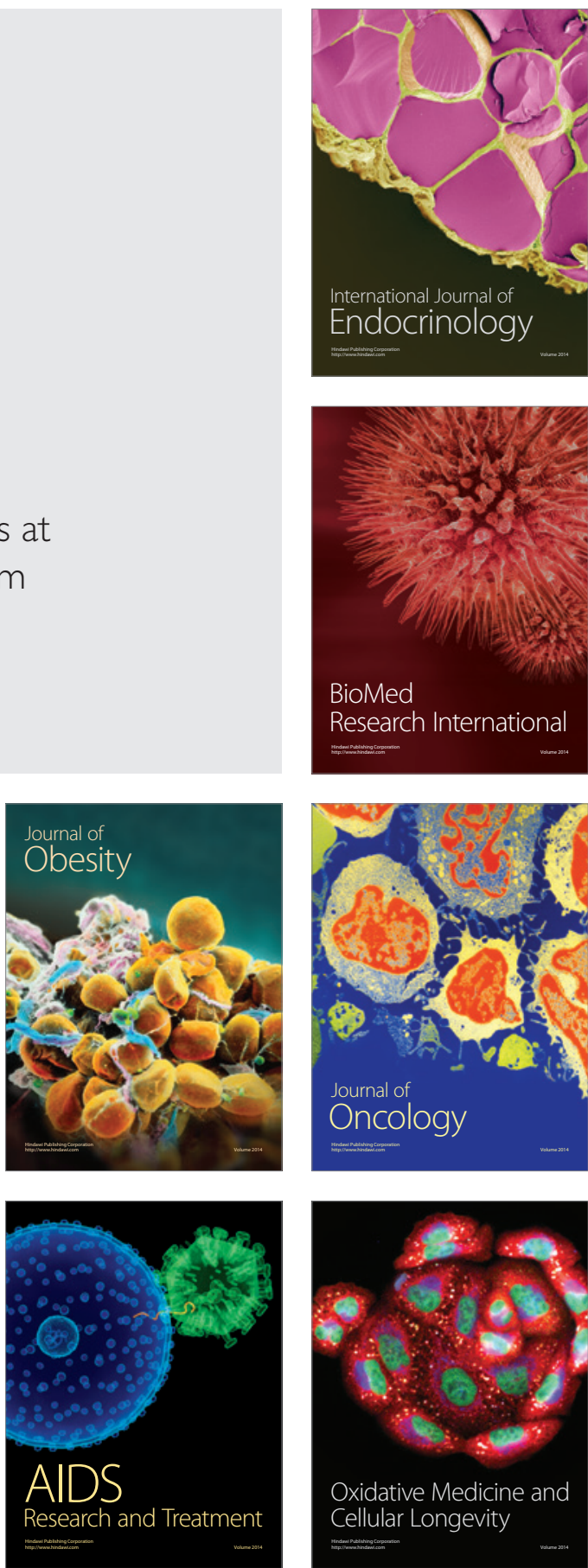